\title{
Editorial
}

\section{Wie Zeitschriften sich entwickeln}

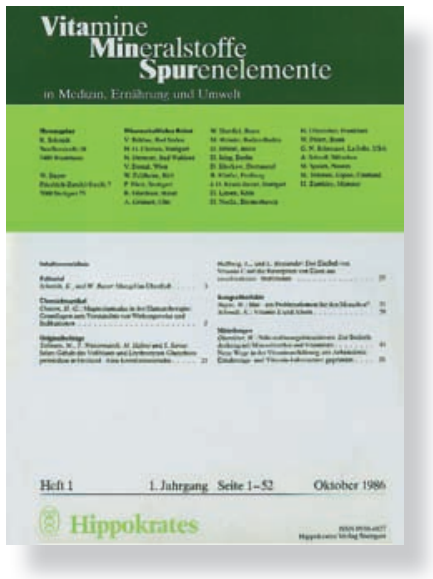

Titel der ersten Ausgabe. Die Namensänderung in Ernährung \& Medizin erfolgte 2002.
Zeitschriften sind merkwürdige Objekte. Bei manchen kann man voraussehen, wie sie sich entwickeln werden. Bei anderen verschätzt man sich vollständig. Statt finanziell auszutrocknen, gedeihen sie ständig, denn sie leben nicht von Anzeigen, sondern von einem wohlhabenden Sponsor oder von Mitgliedsgeldern einer erfolgreichen Fachgesellschaft. Viele andere krebsen dahin. Das Erreichen eines 25. Jahrgangs gibt Anlass zum Rückblick. Bis zum Ersten Weltkrieg war Deutschland ganz vorne im Zeitschriftengründen. Zeitschriften waren sogar Exportschlager. In der Zeit zwischen den beiden Weltkriegen hatte US-Amerika die Gelegenheit, sich wissenschaftlich zu profilieren. Besonders in der Medizin gelang das ganz erstaunlich. Dazu trugen auch die jüdischen Zwangsemigranten bei. Die Amerikaner entwickelten dabei ein System der Qualitätsmessung von Zeitschriftenartikeln, das auf der Annahme beruht, eine naturwissenschaftliche Arbeit sei umso besser, je häufiger sie in einem bestimmten Zeitraum zitiert wurde. Man fing an, die Literaturverzeichnisse von Arbeiten mit ihren zugehörigen Autoren- und Herkunftshinweisen zu sammeln und auszuwerten. Verblüffendes Resultat: Überall standen die USA an der Spitze, gefolgt von Großbritannien. Kein Wunder. Am einfachsten auszuwerten waren englischsprachige Zeitschriften. Die anderen hatten es vergleichsweise schwer.

Die Wissenschaftler begannen sich früh auf das Zitierthema einzustellen. Sie versuchten ihre Arbeiten dort zu publizieren, wo sie die besten Aussichten auf Zitate fanden: in viel zitierten englischamerikanischen Zeitschriften. Welche Uni, welcher Autor, welches Fachgebiet zitiert wurden, die bekamen bald die meisten Zuschüsse und Sponsorengelder. Das Spiel lohnte sich. Für manche war es sehr nützlich, für viele war es ein fragwürdiger Aufwand. Sicher ist inzwischen, dass das Ranking jeder Art ein politisch bedeutsamer Faktor geworden ist. Und ebenso sicher sind Zeitschriften durch das Ranking vorwiegend trockener und langweilig geworden, blutleer und humorlos.

Was kann man einer kaum international zitierten Zeitschrift wie der „E \& M“ empfehlen? So lange sie die Rückmeldung bekommen, dass die „E \& M“ Lust und Laune beim Lesen erzeugt, haben der Verlag und die Herausgeber einen Trumpf in der Hand. Das Thema Ernährung und Medizin liegt im Trend. Starke Lobbyvertreter stehen dahinter, ärztliche Interessenvertreter ebenfalls, nicht zu vergessen die stattliche Zahl der Ernährungsfachkräfte. Ihnen allen wäre mit einer zu starken wissenschaftlichen Ausrichtung nicht gedient, das wäre zu praxisfern und wenig anwendungsfreundlich. Die gewählte Inhaltsstruktur der „E \& M“ erscheint gerade ausgewogen. Und erfolgreich laufende Pferde sollte man nicht aus dem Rennen nehmen.

\section{Christian Staehr, Stuttgart}

